\title{
Frailty and anesthesia - risks during and post-surgery
}

This article was published in the following Dove Press journal: Local and Regional Anesthesia

\author{
Hui-Shan Lin ${ }^{1,2}$ \\ Rebecca L McBride 2,3 \\ Ruth E Hubbard 1,2 \\ 'Centre for Research in Geriatric \\ Medicine, University of Queensland, \\ Princess Alexandra Hospital, Brisbane, \\ QLD, Australia; ${ }^{2}$ PA-Southside Clinical \\ Unit, School of Clinical Medicine, The \\ University of Queensland, Brisbane, \\ QLD, Australia; ${ }^{3}$ Department of \\ Anaesthesia, Princess Alexandra \\ Hospital, Brisbane, QLD, Australia
}

Correspondence: Hui-Shan Lin Centre for Research in Geriatric Medicine, Building 33, Princess Alexandra Hospital,

Ipswich Road, Woolloongabba, Queensland 4102, Australia

Tel +6I 731767402

Fax+6I 731766945

Email huishan.lin@uq.net.au

\begin{abstract}
Frailty is a state of decreased physiologic reserve and resistance to stressors. Its prevalence increases with age and is estimated to be $26 \%$ in those aged above 85 years. As the population ages, frailty will be increasingly seen in surgical patients receiving anesthesia. Here, we evaluate the instruments which have been developed and validated for measuring frailty in surgical patients and summarize frailty tools used in 110 studies linking frailty status with adverse outcomes post-surgery. Frail older people are vulnerable to geriatric syndromes, and complications such as postoperative cognitive dysfunction and delirium are explored. This review also considers how frailty, with its decline of organ function, affects the metabolism of anesthetic agents and may influence the choice of anesthetic technique in an older person. Optimal perioperative care includes the identification of frailty, a multisystem and multidisciplinary evaluation preoperatively, and discussion of treatment goals and expectations. We conclude with an overview of the emerging evidence that Comprehensive Geriatric Assessment can improve postoperative outcomes and a discussion of the models of care that have been developed to improve preoperative assessment and enhance the postoperative recovery of older surgical patients.
\end{abstract}

Keywords: frail, elderly, surgery, perioperative medicine, adverse outcomes

\section{Introduction}

The population aged 65 years and over worldwide is predicted to reach 1.6 billion by year 2050. ${ }^{1}$ As the population ages, the rate of surgical procedures in older people is rapidly increasing and the demand for surgical services and anesthesia is predicted to grow. ${ }^{2,3}$ In England, 2.5 million people over the age of 75 years underwent surgery between years 2014 and 2015, compared with just under 1.5 million between 2006 and $2007 .{ }^{4}$ Nearly $30 \%$ of these 2.5 million adults were over 85 years old. Similar trends were found in Australia. In years 2012-2013, those aged 65 and older represented a significant proportion of elective and emergency admissions involving surgery. ${ }^{5}$ As an increasing number of older patients undergo surgery, anesthetists will be faced with more frail patients in their daily work.

There is a complex interplay between aging, frailty, and anesthesia. In this review, the importance of frailty in surgical patients and its relationship with geriatric syndromes will be considered. Secondly, how frailty impacts on a person's response to anesthetic agents and how anesthesia impacts on frail patient's recovery from surgery will be presented. Finally, principles of perioperative care for frail patients and strategies for preventing adverse outcomes post-anesthesia will be highlighted. 


\section{Frailty}

Frailty is a term used by laymen and some medical staff to describe an older person who appears weak, unsteady, and underweight. This expression often carries a negative connotation implying some concerns about a person's future outlook. ${ }^{6}$ More recently, frailty has been conceptually defined to describe a state of increased vulnerability, a syndrome of decreased physiologic reserve and resistance to stressors.? Frailty can lead to increased adverse outcomes, such as loss of mobility and independence, triggered by relatively small physical insults, such as a new medication or minor infection. ${ }^{6}$

Frailty increases with age and is prevalent in the population: $4 \%$ in the $59-65$-year-olds, $9 \%$ in the $75-79$-year-olds, and $26 \%$ in the above 85 -year-olds. ${ }^{8}$ A systematic review of 21 community-based cohort studies involving 61,500 older adults found that frailty ranges between $4 \%$ and $59 \%$ with an overall weighted prevalence of $10.7 \%{ }^{8}$

The pathogenesis of frailty is thought to involve maladaptive response to stresses in multiple physiological systems, which leads to a loss of dynamic homeostasis. ${ }^{9}$ The pathological processes hypothesized to be responsible for the development of frailty include chronic inflammation and immune activation, sarcopenia (loss of muscle mass and strength), and age-related changes to the endocrine system, such as a decrease in the sex hormones, higher levels of cortisol, and vitamin D deficiency. ${ }^{10}$ These interact together with risk factors, such as genetic and epigenetic factors, environmental and lifestyle stressors, acute and chronic diseases, to result in the clinical manifestation of frailty and adverse health outcomes. ${ }^{10}$ Frailty in older medical patients has been shown to result in increased mortality, worsened functional status, increased falls, hospitalization, and admission to long-term care facilities. ${ }^{7,11,12}$ Emerging evidence in the last 5-10 years has shown that frailty also leads to increased mortality and morbidity in older surgical patients. ${ }^{13-15}$

Even though frailty is well-recognized, there is no consensus on how it should be measured. Abundant scales and instruments have been researched for identifying and quantifying frailty; however, there is no standard tool for screening frailty in routine clinical practice. To date, clinicians rely on instinct and experience to identify frail patients. This "eyeballing" technique can be subjective with large interobserver variability. ${ }^{16}$ Apart from eyeballing, there are two major conceptual models of frailty which have been proposed and from them stemmed frailty instruments.

In the "phenotype" model described by Fried et al, also known as the Cardiovascular Health Study (CHS) definition, frailty manifests as a decline in lean body mass, strength, endurance, walking performance, and activity level. ${ }^{7}$ The Fried criteria measures frailty out of five points. Frailty is defined as the presence of three or more of the five features of slowness, weakness, exhaustion, weight loss, and low physical activity. Patients who have none of these five features are non-frail, those who have one or two of these features are deemed "pre-frail", and those with three or more are deemed "frail". 7

The other model developed by Rockwood et al from the Canadian Study of Health and Aging is the "cumulative deficit" model. ${ }^{17}$ This model conceptualizes aging as the accumulation of deficits and views frailty as a multidimensional risk state quantified by the number of deficits rather than by the nature of the health problems. ${ }^{17}$ While a deficit alone does not result in an illness, for example, hearing impairment alone, difficulty walking alone, heart disease alone, or polypharmacy alone, an accumulation of multiple deficits result in increasing strain on the system and lead to increased vulnerability for system collapse or system failure. The more deficits an individual accumulates, the more frail the person is. Frailty is measured by totalling the number of deficits present in an individual divided by the total number of deficits measured, expressed as an index between 0 and 1. ${ }^{18}$ The deficits considered are from multiple domains, including comorbidities, medications, physical and cognitive impairments, psychosocial risk factors, and common geriatric syndromes. ${ }^{18} \mathrm{~A}$ higher frailty index (FI) indicates a higher degree of frailty. FI represents a continuum; however, it can also be trichotomized to indicate low, intermediate, and high level of frailty $(\mathrm{FI}<0.25$, FI $\geq 0.25-0.4$, FI $>0.4){ }^{19}$

\section{Frailty and surgery}

A hospitalized older surgical patient is faced with many challenges during their journey through surgery and the recovery period. Apart from surgery itself, fasting, opioid analgesics, anesthetic agents, intraoperative blood loss, postoperative pain, nausea and vomiting, the unfamiliar hospital environment, and immobility during the perioperative period can all be triggers that lead a previously balanced but frail body system to fail. The degree of insult needed to cause a decompensation and subsequent adverse events is inversely related to the degree of frailty. A minor insult may be sufficient to lead to permanent functional decline post-surgery in a very frail patient, whereas a robust older person may need major surgery and several postoperative complications to result in a decline in function.

This is illustrated well in the diagram taken from Desserud et al (Figure 1) ${ }^{15}$ Individual "A" may be a fit individual 


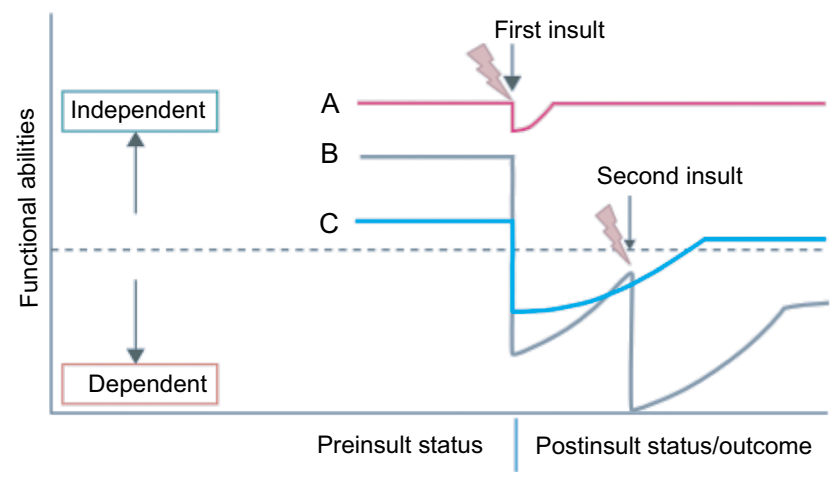

Figure I Role of frailty in recovery from surgery.

Source: Copyright C(1999-2018 John Wiley \& Sons, Inc. Reproduced from Desserud KF, Veen T, Soreide K. Emergency general surgery in the geriatric patient. Br J Surg. 2016; 103(2):e52-61. ${ }^{15}$ from Desserud et al..$^{15}$

who recovers quickly after a minor insult, such as appendicectomy for appendicitis, and who returns to premorbid level of function after surgery. Individual "C" may be a functionally independent person with a moderate degree of frailty, who suffers an intermediate insult, such as an emergency colon cancer surgery, who becomes dependent on care for a period of time postoperatively, who eventually returns to independent living, however, at a reduced long-term function compared with before surgery. Individual " $\mathrm{B}$ " may be an independent individual with a mild degree of frailty, who suffers a major insult, such as strangulated small bowel or perforated peptic ulcer with abdominal sepsis, leading to dependence. If this individual " $\mathrm{B}$ " suffers a second insult, such as postoperative pneumonia, a cardiac event, or an anastomotic leak, further functional decline or even death may result, and recovery to independent living and function would be impossible.

The importance of frailty in surgical patients has gained recognition in the last 5 years, with a rapid emergence of publications on this topic. A search for articles on PubMed published between the years 2013 and end of March 2018 using search terms "frailty" AND "surgical outcome" identified 367 titles, whereas the same search for publications between 2006 and 2010 yielded only 74 titles, showing a fivefold increase in publications in this field in the last 5 years. Examining specifically literature relating frailty measured by multicomponent methods (as opposed to single marker, such as a blood marker or gait speed alone) to adverse postoperative outcomes, there is a surge of publications in year 2016 compared with the years before (Figure 2). In contrast, studies on frailty intervention, although steadily increasing, remain low in number.

In these publications, there are a plethora of instruments which have been studied to measure frailty in surgical patients. In a systematic review of 23 studies examining the relationship between frailty and surgical patients with a mean age of 75 years and above, 21 different instruments were used to measure frailty. ${ }^{20}$ Altogether, 110 studies between years 2007 and 2017 evaluated frailty measured using multidimensional models in relation to adverse outcome in patients of all ages undergoing surgery, and 37 different measurement tools were used. A table summarizing these tools and the types of surgical patients they were applied in is included in the Supplementary material.

Variations of the Fried Criteria or instruments based on Comprehensive Geriatric Assessment (CGA), including the FI, were the most commonly used. Modified FI appeared in the largest number of publications $(n=35)$. This was developed out of the National Surgical Quality Improvement Program (NSQIP) database and consists of a score derived from 11 deficits including functional status, impaired sensorium, cardiovascular comorbidities, COPD, and pneumonia. All these studies were retrospective cohort studies evaluating the association between frailty and various adverse outcomes in a variety of surgical types. Its value in predicting adverse outcome needs to be validated in prospective studies, before it can be used as a screening tool. Scales based on CGA are obtainable from patient interview as well as clinical notes without physical performance-based measures and were used in both acute and elective surgical cohorts. In contrast, the Fried frailty measure required physical performance-based tests and was used exclusively in elective surgical cohorts. Other instruments, such as Multidimensional Frailty Score $^{21}$ and Comprehensive Assessment of Frailty ${ }^{22-24}$ combined aspects of CGA with performance-based tests (e.g., balance assessments, chair rise, stair climb) and medical investigations (e.g., blood test and respiratory function test). Frailty measurement tools need to be not only sensitive and specific at detecting frailty but also time-efficient and brief in order to be routinely applied in preoperative assessment.

\section{Frailty and geriatric syndromes}

Frailty is associated with geriatric syndromes, namely; functional decline, mobility impairment, polypharmacy, delirium, dementia, pressure ulcers, falls, malnutrition, and incontinence, all of which have an impact on postoperative recovery. A prospective cohort study found that 39\% of vascular surgical inpatients were frail and $36 \%$ had geriatric syndromes. ${ }^{25} \mathrm{~A}$ systematic review showed that the incidence of geriatric syndromes in older surgical patients is not well documented, apart from postoperative delirium (POD), with 


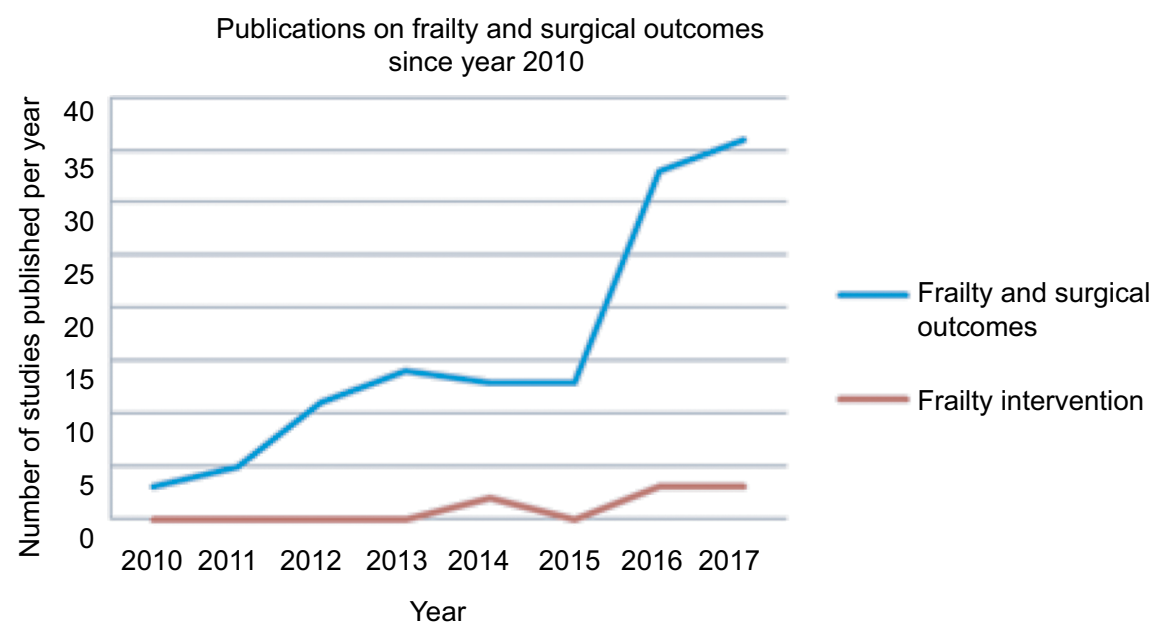

Figure 2 Publications on frailty and surgical outcomes since year 2010.

current evidence showing the incidence of urinary incontinence to be $29 \%$, pressure ulcer to be $3 \%$, malnutrition to be 9-37\%, depression to be $32 \%$, and falls to be $2-6 \%$ among older surgical population. ${ }^{26}$ Preexisting geriatric syndromes make an individual vulnerable to post-surgical complications; on the other hand, non-elective and major surgery increases the likelihood of developing geriatric syndromes during an individual's surgical admission. ${ }^{27}$ Commonly used risk prediction tools such as age, American Society of Anesthesiologists (ASA) physical status, Revised Lee's Cardiac Risk Index, Charlson Comorbidity Index, and exercise tolerance as measured by metabolic equivalent task score (METS), while good at predicting organic specific complications, do not take into account frailty and geriatric syndromes. ${ }^{28}$ These traditional tools are unable to measure vulnerability and decreased reserve of an older adult, which has impact on functional recovery, length of stay, and need for institutionalization post-surgery. ${ }^{28}$ Hence, assessment and identification of frailty and geriatric syndromes in the preoperative setting is important in predicting adverse outcomes in combination with traditional risk tools.

\section{Frailty and pharmacological considerations for anesthesia}

Frailty is the accumulation of deficits across multiple organ systems leading to physiological decline and subsequently alterations in pharmacokinetics and pharmacodynamics. These issues are further compounded by co-morbidity, organ dysfunction, and polypharmacy. A review of the physiological changes with aging and subsequent frailty must be considered by the perioperative team and care must be individualized for the deficits of the patient.

The body composition of an elderly patient is characterized by a decrease in muscle mass, an increase in adipose tissue, and a reduction in total body water. Subsequently, lipophilic drugs have a larger volume of distribution with a potentially longer duration of action, ${ }^{29}$ whereas hydrophilic drugs will have a higher peak plasma concentration due to the reduction in the central compartment. The decrease in muscle mass often means that declining renal function is not reflected precisely by the serum creatinine. Furthermore, aging reduces renal mass, the speed of renal excretion of drugs, and patients have increased sensitivity to drugs with potential for renal toxicity. ${ }^{30}$ Frailty is associated with a reduction in estimated glomerular filtration rate. In pre-dialysis patients with chronic kidney disease, frailty conferred a negative prognostic survival factor. ${ }^{31}$ Drug metabolism is also typically altered in the older population due to reduced hepatic blood flow and a reduction in the activity of the cytochrome $\mathrm{P} 450$ system. Consequently, drugs are less effectively cleared by Phase I reactions, although Phase II reactions are not affected. ${ }^{29}$

Physiological changes to the cardiovascular system with aging include progressive stiffening of both the myocardium and the vasculature. ${ }^{29}$ Consequently, diastolic dysfunction and hypertension are common with increasing age. Cardiac failure is a risk factor for major adverse cardiac events in the perioperative period and is prevalent in up to $75 \%$ of frail patients. ${ }^{32}$ Furthermore, frailty is associated with cardiac autonomic dysfunction, ${ }^{33}$ the impact of which can result in blood pressure lability, particularly profound, prolonged 
hypotension in response to the administration of anesthesia, particularly in the setting of hypovolemia which is often encountered in the perioperative period. ${ }^{29,30}$

Postoperative pulmonary complications account for $40 \%$ of deaths in the older population. ${ }^{29}$ Age confers a progressive loss of lung parenchyma elasticity, a reduction in functional alveolar surface area and reduced respiratory muscle strength. These changes cause an increase in ventilation/ perfusion mismatch and increase the risk of both hypoxemia and atelectasis. Furthermore, a reduction in airway reflexes, particularly in the setting of residual weakness, from drugs such as neuromuscular blocking agents, opioids, or sedatives, may increase the risk of aspiration. ${ }^{29,30}$

\section{Anesthesia and cognition}

Of the geriatric syndromes in older surgical patients, disturbances to cognition in the perioperative period are the most well studied. These disturbances in cognition have been termed postoperative cognitive dysfunction (POCD) and postoperative delirium (POD) which are important in prognosticating an older patient's recovery from surgery. Frail patients are more likely to have pre-existing cognitive impairment with reduced cognitive reserve; hence, they are the most vulnerable to POCD and POD.

Postoperative cognitive dysfunction (POCD) broadly refers to a deterioration in cognition temporally associated with surgery. Particularly, POCD refers to reduction from baseline in multiple domains of cognitive function such as attention, concentration, working memory, executive function, processing speed, set shifting, verbal fluency, and/or visual spatial performance. The diagnostic criteria of POCD are currently debated and rely upon neuropsychological testing. Its prevalence is reported to be $10-30 \%$ in patients when evaluated 1 month after cardiac surgery, and $26 \%$ at 1 month and $10 \%$ at 3 months after non-cardiac surgery. ${ }^{34,35}$ While POCD is a reversible condition in the majority of cases, it persists in about $1 \%$ of cases. ${ }^{36}$ Newman et al reported that POCD at hospital discharge was associated with an increased risk for cognitive decline 5 years after cardiac surgery; however, this result did not account for the cognitive decline which occurs with the aging process. ${ }^{37}$ Two other studies have since compared cognitive decline in patients with coronary artery disease who underwent coronary artery bypass surgery with those who had conservative management and found that the former group did not suffer more cognitive decline than the latter, after adjusting for age, sex, education, and baseline comorbidities. ${ }^{38,39}$ Whether POCD negatively impacts on long-term cognition is yet to be further elucidated.
POD on the other hand has a clear clinical diagnostic criteria and is well described to be associated with adverse outcomes as well as future short- and long-term cognitive decline. ${ }^{40}$ Delirium is characterized by an acute fluctuating course of cognition and consciousness, inattention, disorganized thinking, and perceptual disturbances, not explained by pre-existing or evolving dementia, as defined by the Diagnostic and Statistical Manual of Mental Disorders (5th edition). ${ }^{41}$ Its prevalence is around $30-62 \%$ in surgical patients, ${ }^{41}$ and incidence is around $36 \% .{ }^{42}$ The pathogenesis of POD is complicated and not completely understood. POD has been postulated to be caused by a central cholinergic deficit, ${ }^{43}$ which may have resulted from a combination of anticholinergic medications used and hypoxic injury of the brain during the perioperative period. This is compounded by the systemic inflammation caused by surgery, releasing pro-inflammatory cytokines IL1b and tumor necrosis factor $\alpha$, activating central nervous system microglia which further release pro-inflammatory cytokines which in turn impair acetylcholine synthesis. ${ }^{44,45}$ A patient with reduced cognitive reserve preoperatively would not be able to compensate for these neurochemical changes and when the homeostasis could no longer be maintained, clinical delirium will manifest.

POD leads to increased dependence on care, worse quality of life, higher health care cost, and increased mortality; ${ }^{46,47}$ even 5-year mortality was reported by one study to be increased by as much as 7.35-fold (95\% CI 1.49-36.18) after adjusting for confounders. ${ }^{48}$ Even though delirium is not routinely viewed as a complication after surgery, its impact at the population level is greater than the routinely collected major postoperative complications (life threatening or life altering events based on Accordion Severity grade 2 or greater), contributing not only to prolonged length of stay but also to institutional discharge and 30-day readmission. ${ }^{49}$ Risk factors for POD in vascular surgical patients have been identified to be age, history of dementia, ASA score $>2$, renal failure, previous stroke, history of neurologic condition, male gender, intraoperative blood loss, longer hospital stay, and the need of intensive care, ${ }^{50,51}$ while those in hip fracture cohort are polypharmacy and preoperative indwelling catheter insertion. ${ }^{52}$

\section{Frailty and adverse outcomes}

Frailty has long been shown in medical patients to lead to adverse outcome. The association between frailty and adverse outcomes in surgical patients has gained recognition only in the last few years. A recent systematic review ${ }^{20}$ summarized 
various adverse outcomes studied in relation to frailty in patients with a mean age of 75 years and above (Table 1). The adverse outcomes of interest have been largely focused on mortality and postoperative complications in the surgical literature. For example, 10 out of 10 studies evaluating the relationship between frailty and increased 12-month mortality found a significant relationship with frailty, with odds ratios ranging from 1.1 to $4.97 .{ }^{20}$ Similarly, frailty was found to be associated with shorter (30 days and 90 days) and longer-term ( 2 and 5 years) mortality. This association was found regardless of the instruments used to measure frailty and irrespective of the type of surgery performed.

Postoperative complications evaluated in the current literature are commonly graded by the Clavien-Dindo severity classification $^{53}$ or a predefined set of conditions. Out of nine papers evaluating frailty and postoperative complications,

Table I Adverse outcomes in association to frailty examined in studies including participants with a mean age of $>75$ years

\begin{tabular}{|c|c|c|c|c|c|c|c|c|c|c|c|}
\hline \multirow[t]{2}{*}{ Outcome } & \multicolumn{11}{|c|}{ Number of studies } \\
\hline & & I & 2 & 3 & 4 & 5 & 6 & 7 & 8 & 9 & 10 \\
\hline \multicolumn{12}{|l|}{ Mortality } \\
\hline I-year mortality & Quality & Q1 ${ }^{85}$ & QI ${ }^{21}$ & Q1 ${ }^{86}$ & Q1 ${ }^{87}$ & QI ${ }^{88}$ & Q2 ${ }^{89}$ & Q2 ${ }^{90}$ & Q2 ${ }^{23}$ & Q2 ${ }^{24}$ & Q2 ${ }^{91}$ \\
\hline $\mathrm{n}=10$ & $\mathrm{~N}$ sample & 100 & 275 & 244 & 12979 & 218 & 159 & 30 & 213 & 450 & 410 \\
\hline 2-year mortality & Quality & Q1 ${ }^{88}$ & & & & & & & & & \\
\hline $\mathrm{n}=\mathrm{l}$ & $\mathrm{N}$ sample & 218 & & & & & & & & & \\
\hline 5-year mortality & Quality & Q1 ${ }^{92}$ & & & & & & & & & \\
\hline$n=1$ & $\mathrm{~N}$ sample & 178 & & & & & & & & & \\
\hline 30-day mortality & Quality & QI ${ }^{85}$ & Q1 93 & Q1 94 & Q1 97 & $\mathrm{Q} 2{ }^{22}$ & Q1 98 & & & & \\
\hline $\mathrm{n}=6$ & $\mathrm{~N}$ sample & 100 & 176 & 184 & 178 & 400 & 325 & & & & \\
\hline 90-day mortality & Quality & Q1 ${ }^{87}$ & QI ${ }^{98}$ & & & & & & & & \\
\hline $\mathrm{n}=2$ & $\mathrm{~N}$ sample & 12979 & 325 & & & & & & & & \\
\hline \multicolumn{12}{|c|}{ Post-operative complications } \\
\hline Non-routine recovery & Quality & Q1 99 & Q1 ${ }^{94}$ & Q2 ${ }^{89}$ & Q2 ${ }^{100}$ & Q1 101 & Q1 ${ }^{21}$ & Q1 102 & QI ${ }^{103}$ & $\mathrm{Q}^{86}$ & \\
\hline$n=10$ & $\mathrm{~N}$ sample & 178 & 184 & 159 & 83 & 152 & 275 & 125 & 35 & 244 & \\
\hline Need for resuscitation & Quality & Q2 ${ }^{23}$ & & & & & & & & & \\
\hline $\mathrm{n}=\mathrm{I}$ & $\mathrm{N}$ sample & 213 & & & & & & & & & \\
\hline Delirium & Quality & Q। 103 & & & & & & & & & \\
\hline $\mathrm{n}=\mathrm{I}$ & $\mathrm{N}$ sample & 35 & & & & & & & & & \\
\hline MACCE & Quality & Q1 ${ }^{85}$ & Q2 ${ }^{23}$ & Q2 ${ }^{90}$ & & & & & & & \\
\hline$n=3$ & $\mathrm{~N}$ sample & 100 & 213 & 30 & & & & & & & \\
\hline \multicolumn{12}{|l|}{ Discharge } \\
\hline Length of stay & Quality & QI 97 & QI ${ }^{103}$ & QI 98 & Q2 ${ }^{91}$ & $\mathrm{Q} 2{ }^{89}$ & Q1 102 & & & & \\
\hline $\mathrm{n}=6$ & $\mathrm{~N}$ sample & 178 & 35 & 325 & 410 & 159 & 125 & & & & \\
\hline Discharge to institution & Quality & QI ${ }^{21}$ & Q2 91 & & & & & & & & \\
\hline $\mathrm{n}=3$ & $\mathrm{~N}$ sample & 275 & 410 & & & & & & & & \\
\hline Functional decline & Quality & Q। 102 & & & & & & & & & \\
\hline $\mathrm{n}=\mathrm{I}$ & $\mathrm{N}$ sample & 125 & & & & & & & & & \\
\hline \multicolumn{12}{|l|}{ Post-discharge } \\
\hline Readmission rate: & Quality & Q2 ${ }^{91}$ & Q1 98 & & & & & & & & \\
\hline I year & $\mathrm{N}$ sample & 410 & 325 & & & & & & & & \\
\hline \multicolumn{12}{|l|}{$n=2$} \\
\hline Functional decline & Quality & QI ${ }^{104}$ & Q2 ${ }^{105}$ & & & & & & & & \\
\hline \multirow[t]{3}{*}{$\mathrm{n}=2$} & $\mathrm{~N}$ sample & 119 & 84 & & & & & & & & \\
\hline & & at 6 & $16-28$ & & & & & & & & \\
\hline & & months & months & & & & & & & & \\
\hline Quality of life: & Quality & Q1 ${ }^{86}$ & & & & & & & & & \\
\hline 6 months, I year & $\mathrm{N}$ sample & 244 & & & & & & & & & \\
\hline
\end{tabular}

Note: Dark and light shades represent significant and non-significant associations, respectively.

Abbreviations: P, prospective study; R, retrospective study; QI, quartile one quality assessment; Q2, quartile two quality assessment; MACCE, Major Cardiac and Cerebral Adverse Events; $n$, number of studies. 
only five reported significant association, with odds ratios ranging from 1.5 to $4.8 .^{20}$ Other outcome measures such as length of stay, quality of life, delirium, functional decline, and discharge to a residential care facility have been less studied; however, the few studies that did include these outcomes still found significant associations between those and frailty. ${ }^{20}$

Due to the heterogeneity of the study populations, types of surgery performed, and the methods of frailty measures, meta-analysis for evaluating the overall effect size of frailty on adverse outcomes has not been available in current systematic reviews. This is a gap that future studies can explore. Furthermore, adverse outcomes which are important to older people, such as quality of life, functional decline/increased dependency, and delirium (which will impact on long-term cognition post-surgery) in relation to frailty, need further exploration and validation. Several studies also suggested that frailty is a better predictor of mortality and morbidity than age, ASA, or comorbidities alone. How frailty compares with and complements traditional surgical risk assessment tools also needs further research.

\section{Frailty and anesthetic techniques}

Whether anesthetic technique has a significant impact on outcomes in frail surgical patients is unclear. The appropriate anesthetic technique used for the performance of surgery that is suitable to either regional or general anesthesia (GA) is vigorously debated. The anesthetic technique chosen for the management of a patient will depend on both the surgical requirements and patient comorbidities. Some patients, due to medications, comorbidities, or preference, are not amenable to regional anesthesia. Similarly, the type of surgery or expected duration may prevent the use, at least exclusively, of regional anesthesia. Regional anesthesia techniques include neuraxial blockade using either spinal or epidural anesthesia or a combination of the two and peripheral nerve blocks. Any of these techniques may be supplemented with a variety of analgesic agents, sedatives, or GA.

Theoretical benefits to regional anesthesia include the avoidance of exposure to GA and a reduction in airway and pulmonary complications ${ }^{54}$ which frail patients are susceptible to. Neuraxial anesthesia is associated with hypotension and potential for complications including epidural hematoma, infection, and post-dural puncture headache. ${ }^{55}$ The patient also has to be willing to undergo regional anesthesia. The techniques used for sedation used in conjunction with regional anesthesia are highly variable, and its use may ameliorate the benefits of avoidance of GA. A meta-analysis considering the impact of anesthetic technique on postopera- tive cognitive dysfunction and delirium failed to demonstrate a statistically significant difference between regional and GA (odds ratio for POD/POCD in GA versus non-GA was $0.88,95 \%$ CI $0.51-1.51$ ), however, GA was marginally nonsignificantly associated with POCD (odds ratio of $1.34,95 \%$ CI $0.93-1.95) .{ }^{56}$ Furthermore, regional anesthesia is likely to be beneficial for analgesia, the extent of which depends on the type of block used, and may potentially reduce the side effects of systemic analgesia.

Despite the increasing interest in frailty as a risk factor for perioperative morbidity and mortality, there remains a relative paucity of well-designed and recent studies specifically comparing different modern anesthetic techniques in the frail population. Hip fracture remains the most well-studied topic in the frail population, and the variation in both publications and practice illustrates the substantial uncertainty about the implications of the anesthetic..$^{57,58}$ The National Hip Fracture Database in the United Kingdom recorded that $~ 50 \%$ received general anesthetic, whereas around 44\% received spinal anesthesia. ${ }^{59,60}$ It was noted that there was a great variation between institutions, with some hospitals administering spinal anesthetics in $\sim 80 \%$ of cases, whereas in others, the administration rate was as low as $10 \% .{ }^{59,60}$ Rates of spinal anesthesia for hip fracture in the United States is thought to be approximately half of the rates observed in UK. ${ }^{57}$ Similar variation in practice is seen in Australia and New Zealand hip fracture surgery cases, with the rate of GA averaging 66\% (Australia) and 64\% (NZ), and the range being 20-97\% between different hospitals. ${ }^{61}$ The most recent Cochrane review further supports the suitability of multiple techniques for the management of neck of femur fracture, indicating there is no difference in either mortality or morbidity between regional techniques and GA. ${ }^{62}$

More recent analysis of retrospective cohort data from the American College of Surgeons' NSQIP database has indicated that regional anesthesia (spinal or regional blockade) had more perioperative complications when compared with GA. ${ }^{63}$ The regional versus GA for promoting independence after hip fracture (REGAIN) trial is a large international, multicenter trial which is currently in progress. Its results expected to be released in 2019 will hopefully clarify the impact of anesthetic technique on hip fracture patients. ${ }^{54} \mathrm{At}$ this point, there is neither clear evidence nor a consensus in expert opinion. Therefore, the anesthetic management should be carefully selected based on expertise and patient's preference.

Frail patients are among the most vulnerable to the physiological changes in the perioperative period. Care- 
ful consideration of the issues associated with frailty and the development of a frailty-specific anesthetic plan to minimize these changes should be advocated. ${ }^{64}$ Therefore, increased monitoring such as Bispectal Index (BIS) or intraarterial blood pressure monitoring should be considered on an individual patient basis. The depth of anesthesia is traditionally based on population estimates of the amount of drug required to prevent recall and movement. Limiting excessive exposure to anesthetic agents was hypothesized to minimize the rates of postoperative cognitive dysfunction and delirium. The use of processed electroencephalogram to measure the depth of anesthesia has attempted to elucidate the optimal dose for an individual patient. A recent review article reported an association between increased depth of anesthesia as indicated by a BIS of less than 45 and higher rate of death, myocardial infarction, and postoperative cognitive dysfunction. ${ }^{65}$ Unfortunately, the majority of studies to date have utilized the BIS monitor to measure the depth of anesthesia, and reached this conclusion following the post hoc analyses of studies that were intended for other purposes, without controlling for intraoperative hypotension. The Balanced Anesthesia Study, a prospective randomized controlled study in progress, aims to clarify the causative relationship of low BIS with mortality, controlling for hypotension. ${ }^{66}$ Certainly "triple low state" where the BIS, volatile anesthetic minimum alveolar concentration equivalent, and mean arterial blood pressure are low is known to increase the risks of postoperative mortality. ${ }^{67}$ These potentially modifiable factors should be avoided in frail patients undergoing anesthesia.

\section{Frailty interventions}

Despite the strong evidence that frailty in surgical patients leads to poorer postoperative outcomes, there is still a lack of a unifying tool which is time-efficient and practical for measuring frailty. This is a barrier for its current usage in surgical pre-admission clinics. Detection of frailty at baseline preoperatively could aid in identification of high-risk patients with potential poor outcomes. Instituting supportive and preventative measures during their hospital admission may optimize their outcomes.

Frail elderly surgical patients would benefit from early recognition and treatment of surgical complications, postoperative infections, monitoring of adequate hydration and nutrition, and early mobilization and rehabilitation to prevent deconditioning. ${ }^{15}$ Earlier recognition of complications is likely to reduce the chance of failure to rescue patients and improve outcomes. ${ }^{68}$ Evaluation of cognition, which is cur- rently not a routine practice but a part of frailty assessment, could also lead to preventive measures to decrease the incidence of POD. Surgical patients at high risk of POD would benefit from multidisciplinary team input, early mobilization, sleep hygiene, avoidance of restraints, adequate nutrition, fluids, oxygen, and adequate pain control while minimizing the use of opioids. ${ }^{69}$ Many of these strategies have been incorporated into the optimal perioperative management of the geriatric patient practice guidelines from the American College of Surgeons. ${ }^{70}$

Older people presenting with hip fractures are some of the frailest surgical patients. ${ }^{71}$ The ortho-geriatric care model where patients are comanaged by geriatricians and orthopedic surgeons has led to reduced mortality ${ }^{72}$ and has now become the standard of care in most first-world countries. This shared care model involving multidisciplinary teams throughout the perioperative period to enhance optimal timing and patient selection for surgery and improved surveillance to enhance postoperative care has been shown to both reduce complications and improve satisfaction with patient care. $^{68}$

Several service improvement models have also been developed and trialed in elective surgical patients, such as the "POPS" (Proactive care of Older People undergoing Surgery) ${ }^{73}$ and the PSH (Perioperative Surgical Home $)^{74,75}$ with a focus on coordinated, multidisciplinary, and patient-centered care. The latter model, however, is led by anesthetists and has not yet incorporated geriatric assessment. Enhanced Recovery After Surgery (ERAS) protocols have several components addressing frailty, such as optimization of nutrition preoperatively and early mobilization postoperatively; however, they may not address all components of frailty such as cognitive impairment. ${ }^{76}$ In a systematic review, preoperative CGA has also been shown to improve postoperative outcomes such as complication rate and length of stay. ${ }^{77} \mathrm{~A}$ recent randomized controlled trial of elective vascular surgical patients aged 65 years or older showed that preoperative CGA and optimization was associated with a shorter length of hospital stay, fewer complications, and were less likely to be discharged to a higher level of care. ${ }^{78}$ Similarly, a cluster-randomized controlled trial of a Hospital Elder Life Program orientating communication, nutritional assistance, and early mobilization in 577 elective abdominal surgical patients showed reduced rates of delirium and length of stay. ${ }^{79}$ Another prospective cohort quality improvement project involving 9153 patients showed that widespread frailty screening preoperatively reduces mortality. ${ }^{64}$ 
For elective surgical patients, prehabilitation or reversal of frailty may make them a fitter candidate for surgery. Studies have suggested that prehabilitation before surgery with a multimodal program consisting of exercise training and nutritional and psychological support may lead to better functional capacity postoperatively than rehabilitation after surgery. ${ }^{80,81}$ A systematic review of preoperative exercise intervention in cancer patients showed significant improvement in the rate of incontinence, functional walking capacity, and cardiorespiratory fitness. ${ }^{82}$ A pilot study of health coaching and wellness plans through the Community Actions and Resources Empowering Seniors model showed that this initiative decreases frailty status in the primary care setting. ${ }^{83}$ In a very small cohort study of frail lung cancer patients $(\mathrm{n}=14)$, preoperative high-intensity training program could reduce postoperative complications. ${ }^{84}$ In frail pretransplant patients, prehabilitation through fitness-based interventions with wearable fitness tracking devices can mitigate frailty and decrease length of hospital stay and postoperative complications. ${ }^{85}$ More large-scale intervention studies on frailty in preoperative patients are needed to confirm its effectiveness in reducing morbidity and mortality.

Considering the usage of regional anesthesia for postoperative analgesia, thus reducing the requirement of systemic opiates, is another way of reducing postoperative complications in frail patients. The techniques offered for analgesia depend on whether the operation is amenable to regional anesthesia, a lack of contraindications, and the likely duration of significant pain. The options include central neuraxial blockade using either an epidural with a catheter or spinal anesthesia, which is limited to a single dose, or the use of peripheral nerve blocks performed either as a single shot or with the insertion of a catheter and the administration of local anesthesia by either infusion or boluses.

Epidural anesthesia is undoubtedly the most effective form of pain relief and reduces opioid consumption. ${ }^{86} \mathrm{~A}$ recent meta-analysis concluded that epidural in addition to GA reduced both postoperative mortality and morbidity; however, there were higher rates of hypotension and urinary retention and a technical failure rate of $6.1 \%{ }^{87}$ The mean age of the participants in the 125 trials included in this metaanalysis was not reported, and it is likely that they represent the general population undergoing surgery, which may benefit less from regional anesthesia than those who are frail. Epidural anesthesia is likely to reduce the incidence of paralytic ileus in the general population following open abdominal surgery, although the quality of evidence is considered low. ${ }^{88}$ Patients are receiving more modern techniques such as minimally invasive surgery which may lessen the potential benefits of an epidural. Practically, increasing age decreases the volume of local anesthetic required and increases the risk of motor blockade. ${ }^{89}$ The benefit of epidural anesthesia needs to be balanced with its need for an indwelling urinary catheter, intravenous access and potential hypotension and motor blockade, therefore reducing early mobilization, which contradicts modern ERAS protocols, may increase rates of $P O D$.

The use of neuraxial opioids, specifically intrathecal morphine, in the older population may provide more effective pain relief than systemic opioids. ${ }^{89}$ However, intrathecal morphine administration may be complicated by sedation and pruritus. Respiratory depression is one of the most feared complications, and it may mean that patients who develop pain after the administration of intrathecal morphine either receive inadequate analgesia or require higher levels of monitoring, such as in intensive care, to manage the potential for delayed respiratory depression.

Peripheral nerve blockade involves the placement of local anesthetic in proximity to a nerve and may be used as an adjunct to multimodal analgesia or for anesthesia. There are few contraindications in older patients who will likely benefit from improved analgesia with peripheral nerve blockade and reduction in systemic opioids. ${ }^{89}$ Modern techniques commonly use ultrasound to improve visualization and success rates. The risk of serious complications is low, although dependent on block type and dosing of local anesthetic. A Cochrane Database of Systematic reviews on peripheral nerve block for hip fractures reports improved analgesia and reduced time to mobilization; there was inadequate power to comment on rates of acute confusional state, myocardial infarction, and death. ${ }^{90}$

\section{Principles of perioperative care in frail patients}

The preoperative assessment is the foundation for evaluating a patient prior to surgery and should establish the indications for surgery and assess the patient's comorbidities, enabling risk stratification. The American Geriatric Society recommends that the preoperative assessment of older patients include a multisystem, multidisciplinary evaluation which extends beyond the standard preoperative assessment to include treatment goals and expectations. ${ }^{91}$ In this population, quality of life, treatment expectations, and contemplation of end-of-life preferences should be considered, particularly in the context of increased mortality and morbidity and discharge to residential care in the frailer population. ${ }^{33}$ 
Patients should be encouraged to bring their usual sensory aids, such as glasses and hearing aids, to the hospital. The preoperative clinic is a timely opportunity for optimal medical management and consideration of polypharmacy. Drugs such as benzodiazepines, antihistamines, and tricyclic antidepressants may increase the risk of over-sedation, falls, and orthostatic hypotension. ${ }^{92}$ The potential benefits of continuing these medications may outweigh the risks. A thorough assessment of each patient will enable patient-focused care for the perioperative period.

The assessment of neurocognitive status in the older population should form an essential part of the preoperative assessment. Identifying patients with evidence of cognitive impairment has implications for reliability of history, decision-making capacity, and informed consent. ${ }^{91}$ Impaired cognition may impact on the potential appropriate options for both appropriate anesthesia and analgesia. Furthermore, polypharmacy increases the risk of drug interactions which can have a significant impact on postoperative recovery. Older patients have increased sensitivity to both opioids and anesthetic agents, and therefore careful titration is essential. ${ }^{29}$ Identifying patients with high risks for POD is integral to good perioperative care and preventative measures, as reducing rates of POD play a major role in reducing the morbidity and mortality of surgical patients.

Functional status is used in the cardiac risk stratification of patients as part of both the American College of Cardiology/ American Heart Association and European guidelines. ${ }^{93,94}$ Duke's Activity Status Index is used to estimate more formally a patient's functional capacity in terms of metabolic equivalents. These estimates are essential for perioperative planning of investigations in non-emergency circumstances, if it will impact on clinical care. However, functional status in the elderly can be difficult to measure or estimate due to the impact of comorbidities, disability, and frailty. Functional impairment is associated with increased morbidity, such as surgical site infection, delirium, and pneumonia, and increased mortality. ${ }^{92}$

Identifying frailty and geriatric syndromes is also increasingly considered as part of the preoperative assessment and perioperative care. Knowing surgical patients' frailty status is highly essential in communication with patients, families, and for obtaining informed consent. Explaining the higher risk of postoperative complications will preempt potential adverse outcomes and give patients and families realistic expectations after surgery. Those who are extremely frail may accept the high risk of morbidity and mortality while undergoing palliative surgery with the goal of improved quality of life; however, they may wish to opt for non-operative management over a curative surgery, which may treat a disease but not necessarily improve life quality after surgery. Discharge to a residential care facility and inability to maintain independence post-surgery can be a significant and non-acceptable adverse outcome for many older patients; in some, this may be considered worse than death. In these patients, the decision to receive surgical treatment may change if the risk of institutionalization is disclosed and discussed preoperatively. Finally, for those with a high level of frailty and perioperative risk, the option for nonoperative management of a surgical condition should be given and its consequences explained, with early consultation from palliative care physicians, to enable frail patients to make informed choices about their surgery.

\section{Conclusion}

Frailty is important to recognize in older surgical patients and has been measured by myriads of instruments. The two main methods of measuring frailty are the phenotypic and the cumulative deficit model. Frailty is associated with significant postoperative mortality and morbidity and may affect the choice of anesthetic technique and analgesics used perioperatively. The use of GA compared to regional anesthesia is fiercely debated in the literature and in clinical practice without a clear consensus. A perioperative management plan for the frail patient should address their individual deficits, likely complications, and surgical goals. Potential benefits of regional anesthesia for both surgery and postoperative analgesia should be considered, and the anesthetic technique chosen must be individualized to the patient. There is emerging evidence that CGA in the preoperative setting can improve outcomes. Prehabilitation is a new concept which is now increasingly used to intervene on frail patients and optimize their physical function and psychological state before elective surgery. Collaboration between the treating teams and the patient is pertinent for the development of a perioperative management plan that extends from preoperative planning, throughout the acute medical admission through to rehabilitation and discharge planning. The time has come for systematic implementation of frailty assessment in older surgical patients and pathways for individualized management, including discussion of likelihood of long-term adverse outcomes and the option of nonoperative treatment.

\section{Author contributions}

$\mathrm{H}-\mathrm{SL}$ was responsible for the writing of the majority of the first draft and the submission of the manuscript. RLMB was responsible for the writing of three major sections of the 
manuscript. REH contributed to the conceptualization, structure, and editing of the manuscript. All authors contributed toward data analysis, drafting and revising the paper and agree to be accountable for all aspects of the work.

\section{Disclosure}

The authors report no conflicts of interest in this work.

\section{References}

1. United States Census Bureau [homepage on the Internet]. U.S. Population Aging Slower than Other Countries, Census Bureau Reports. 2016. Available from: http://www.census.gov/newsroom/press-releases/2016/ cb16-54.html?intcmp=sd|pop|03282016. Accessed April 26, 2018.

2. Dall TM, Gallo PD, Chakrabarti R, West T, Semilla AP, Storm MV. An aging population and growing disease burden will require a large and specialized health care workforce by 2025. Health Aff (Millwood). 2013;32(11):2013-2020.

3. Etzioni DA, Liu JH, Maggard MA, Ko CY. The aging population and its impact on the surgery workforce. Ann Surg. 2003;238(2): 170-177.

4. NHS Digital. Hospital Episode Statistics (HES). Available from: https:/digital.nhs.uk/data-and-information/data-tools-and-services/ data-services/hospital-episode-statistics. Accessed April 26, 2018.

5. Australian Institute of Health and Welfare [homepage on the Internet]. Australian Hospital Statistics 2012-13. Health Services Series: No. 54 Canberra: Australian Institute of Health and Welfare; 2014. Available from: http://www.aihw.gov.au/publication-detail/?id=60129546922. Accessed April 26, 2018.

6. Clegg A, Young J, Iliffe S, Rikkert MO, Rockwood K. Frailty in elderly people. Lancet. 2013;381(9868):752-762.

7. Fried LP, Tangen CM, Walston J, et al. Frailty in older adults: evidence for a phenotype. J Gerontol A Biol Sci Med Sci. 2001;56(3):M146-M156.

8. Collard RM, Boter H, Schoevers RA, Oude Voshaar RC. Prevalence of frailty in community-dwelling older persons: a systematic review. $J$ Am Geriatr Soc. 2012;60(8):1487-1492.

9. Fried LP, Xue QL, Cappola AR, et al. Nonlinear multisystem physiological dysregulation associated with frailty in older women: implications for etiology and treatment. J Gerontol A Biol Sci Med Sci. 2009;64(10):1049-1057.

10. Chen X, Mao G, Leng SX. Frailty syndrome: an overview. Clin Interv Aging. 2014;9:433-441.

11. Rockwood K, Howlett SE, MacKnight C, et al. Prevalence, attributes, and outcomes of fitness and frailty in community-dwelling older adults: report from the Canadian study of health and aging. J Gerontol A Biol Sci Med Sci. 2004;59(12):1310-1317.

12. Woods NF, LaCroix AZ, Gray SL, et al. Frailty: emergence and consequences in women aged 65 and older in the Women's Health Initiative Observational Study. JAm Geriatr Soc. 2005;53(8):1321-1330.

13. Sepehri A, Beggs T, Hassan A, et al. The impact of frailty on outcomes after cardiac surgery: a systematic review. J Thorac Cardiovasc Surg. 2014;148(6):3110-3117.

14. Beggs T, Sepehri A, Szwajcer A, Tangri N, Arora RC. Frailty and perioperative outcomes: a narrative review. Can JAnaesth. 2015;62(2):143-157.

15. Desserud KF, Veen T, Soreide K. Emergency general surgery in the geriatric patient. Br J Surg. 2016;103(2):e52-61.

16. Hubbard RE, Story DA. Does frailty lie in the eyes of the beholder? Heart Lung Circ. 2015;24(6):525-526.

17. Rockwood K, Song X, MacKnight C, et al. A global clinical measure of fitness and frailty in elderly people. CMAJ. 2005;173(5):489-495.

18. Rockwood K, Mitnitski A. Frailty in relation to the accumulation of deficits. J Gerontol A Biol Sci Med Sci. 2007;62(7):722-727.

19. Singh I, Gallacher J, Davis K, Johansen A, Eeles E, Hubbard RE. Predictors of adverse outcomes on an acute geriatric rehabilitation ward. Age Ageing. 2012;41(2):242-246.
20. Lin HS, Watts JN, Peel NM, Hubbard RE. Frailty and post-operative outcomes in older surgical patients: a systematic review. BMC Geriatr. 2016;16(1):157.

21. Kim SW, Han HS, Jung HW, et al. Multidimensional frailty score for the prediction of postoperative mortality risk. JAMA Surg. 2014;149(7):633-640.

22. Sundermann S, Dademasch A, Praetorius J, et al. Comprehensive assessment of frailty for elderly high-risk patients undergoing cardiac surgery. Eur J Cardiothorac Surg. 2011;39(1):33-37.

23. Sundermann S, Dademasch A, Rastan A, et al. One-year follow-up of patients undergoing elective cardiac surgery assessed with the Comprehensive Assessment of Frailty test and its simplified form. Interact Cardiovasc Thorac Surg. 2011;13(2):119-123; discussion 123.

24. Sundermann SH, Dademasch A, Seifert B, et al. Frailty is a predictor of short- and mid-term mortality after elective cardiac surgery independently of age. Interact Cardiovasc Thorac Surg. 2014;18(5):580-585.

25. McRae PJ, Walker PJ, Peel NM, et al. Frailty and geriatric syndromes in vascular surgical ward patients. Ann Vasc Surg. 2016;35:9-18.

26. McRae PJ, Mudge AM, Peel NM, Walker PJ. Geriatric syndromes in older surgical patients: a literature review. J Frailty Aging. 2013;2(4):205-210.

27. McRae PJ, Peel NM, Walker PJ, de Looze JW, Mudge AM. Geriatric syndromes in individuals admitted to vascular and urology surgical units. J Am Geriatr Soc. 2014;62(6):1105-1109.

28. Kim S, Brooks AK, Groban L. Preoperative assessment of the older surgical patient: honing in on geriatric syndromes. Clin Interv Aging. 2014;10:13-27.

29. Rana MV, Bonasera LK, Bordelon GJ. Pharmacologic considerations of anesthetic agents in geriatric patients. Anesthesiol Clin. 2017;35(2):259-271.

30. Strom C, Rasmussen LS, Steinmetz J. Practical management of anaesthesia in the elderly. Drugs Aging. 2016;33(11):765-777.

31. Chowdhury R, Peel NM, Krosch M, Hubbard RE. Frailty and chronic kidney disease: a systematic review. Arch Gerontol Geriatr. 2017;68:135-142.

32. Butrous H, Hummel SL. Heart failure in older adults. Can J Cardiol. 2016;32(9):1140-1147.

33. Shem Tov L, Matot I. Frailty and anesthesia. Curr Opin Anaesthesiol. 2017;30(3):409-417.

34. Hogue CW Jr, Palin CA, Arrowsmith JE. Cardiopulmonary bypass management and neurologic outcomes: an evidence-based appraisal of current practices. Anesth Analg. 2006;103(1):21-37.

35. Moller JT, Cluitmans P, Rasmussen LS, et al. Long-term postoperative cognitive dysfunction in the elderly ISPOCD1 study. ISPOCD investigators. International Study of Post-Operative Cognitive Dysfunction. Lancet. 1998;351(9106):857-861.

36. Abildstrom H, Rasmussen LS, Rentowl P, et al. Cognitive dysfunction 1-2 years after non-cardiac surgery in the elderly. ISPOCD group. International Study of Post-Operative Cognitive Dysfunction. Acta Anaesthesiol Scand. 2000;44(10):1246-1251.

37. Newman MF, Kirchner JL, Phillips-Bute B, et al. Longitudinal assessment of neurocognitive function after coronary-artery bypass surgery. N Engl J Med. 2001;344(6):395-402.

38. Selnes OA, Grega MA, Bailey MM, et al. Cognition 6 years after surgical or medical therapy for coronary artery disease. Ann Neurol. 2008;63(5):581-590.

39. van Dijk D, Moons KG, Nathoe HM, et al. Cognitive outcomes five years after not undergoing coronary artery bypass graft surgery. Ann Thorac Surg. 2008;85(1):60-64.

40. Saczynski JS, Marcantonio ER, Quach L, et al. Cognitive trajectories after postoperative delirium. $N$ Engl J Med. 2012;367(1):30-39.

41. APA. Diagnostic and Statistical Manual of Mental Disorders. 5th ed. Arlington, VA: American Psychiatric Publishing; 2013.

42. Hussain M, Berger M, Eckenhoff RG, Seitz DP. General anesthetic and the risk of dementia in elderly patients: current insights. Clin Interv Aging. 2014;9:1619-1628.

43. Koponen HJ. Neurochemistry and delirium. Dement Geriatr Cogn Disord. 1999;10(5):339-341. 
44. Perry VH. The influence of systemic inflammation on inflammation in the brain: implications for chronic neurodegenerative disease. Brain Behav Immun. 2004;18(5):407-413.

45. Hala M. Pathophysiology of postoperative delirium: systemic inflammation as a response to surgical trauma causes diffuse microcirculatory impairment. Med Hypotheses. 2007;68(1):194-196.

46. Sieber FE. Postoperative delirium in the elderly surgical patient. Anesthesiol Clin. 2009;27(3):451-464.

47. Inouye SK. Delirium in older persons. N Engl J Med. 2006;354(11): 1157-1165.

48. Moskowitz EE, Overbey DM, Jones TS, et al. Post-operative delirium is associated with increased 5-year mortality. Am J Surg. 2017;214(6):1036-1038.

49. Gleason LJ, Schmitt EM, Kosar CM, et al. Effect of delirium and other major complications on outcomes after elective surgery in older adults. JAMA Surg. 2015;150(12):1134-1140.

50. Oldroyd C, Scholz AFM, Hinchliffe RJ, McCarthy K, Hewitt J, Quinn TJ. A systematic review and meta-analysis of factors for delirium in vascular surgical patients. J Vasc Surg. 2017;66(4):1269-1279.e1269.

51. Galyfos GC, Geropapas GE, Sianou A, Sigala F, Filis K. Risk factors for postoperative delirium in patients undergoing vascular surgery. $J$ Vasc Surg. 2017;66(3):937-946.

52. Kim JY, Yoo JH, Kim E, et al. Risk factors and clinical outcomes of delirium in osteoporotic hip fractures. J Orthop Surg (Hong Kong). 2017;25(3):2309499017739485.

53. Dindo D, Demartines N, Clavien PA. Classification of surgical complications: a new proposal with evaluation in a cohort of 6336 patients and results of a survey. Ann Surg. 2004;240(2):205-213.

54. Neuman MD, Ellenberg SS, Sieber FE, et al. Regional versus General Anesthesia for Promoting Independence after Hip Fracture (REGAIN): protocol for a pragmatic, international multicentre trial. BMJ Open. 2016;6(11):e013473.

55. Whiting PS, Molina CS, Greenberg SE, Thakore RV, Obremskey WT, Sethi MK. Regional anaesthesia for hip fracture surgery is associated with significantly more peri-operative complications compared with general anaesthesia. Int Orthop. 2015;39(7):1321-1327.

56. Mason SE, Noel-Storr A, Ritchie CW. The impact of general and regional anesthesia on the incidence of post-operative cognitive dysfunction and post-operative delirium: a systematic review with metaanalysis. J Alzheimers Dis. 2010;22(Suppl 3):67-79.

57. Murthy S, Hepner DL, Cooper Z, Bader AM, Neuman MD. Controversies in anaesthesia for noncardiac surgery in older adults. Br JAnaesth. 2015;115(Suppl 2):ii15-ii25.

58. Oliver D, Griffiths R, Roche J, Sohota O. Hip fracture. Clin Evid. 2010;5(1110):1-49.

59. Falls and Fragility Fracture Audit Programme. National Hip Fracture Database (NHFD) Annual Report. 2017. Available from: https://www. nhfd.co.uk/files/2017ReportFiles/NHFD-AnnualReport2017.pdf.

60. Falls and Fragility Fracture Audit Programme. National Hip Fracture Database: Anaesthesia Sprint Audit of Practice (ASAP). 2014. Available from: https://www.nhfd.co.uk/20/hipfracturer.nsf/vwContent/ asapReport/\$file/onlineASAP.pdf.

61. Australia \& New Zealand Hip Fracture Registry. ANZHFR Annual Report. 2017. Available from: http://anzhfr.org/2017-annual-report/.

62. Guay J, Parker MJ, Gajendragadkar PR, Kopp S. Anaesthesia for hip fracture surgery in adults. Cochrane Database Syst Rev. 2016;2:CD000521.

63. Hausman MS Jr, Jewell ES, Engoren M. Regional versus general anesthesia in surgical patients with chronic obstructive pulmonary disease: does avoiding general anesthesia reduce the risk of postoperative complications? Anesth Analg. 2015;120(6):1405-1412.

64. Hall DE, Arya S, Schmid KK, et al. Association of a frailty screening initiative with postoperative survival at 30, 180, and 365 Days. JAMA Surg. 2017;152(3):233-240.

65. Leslie K, Short TG. Anesthetic depth and long-term survival: an update. Can J Anaesth. 2016;63(2):233-240.

66. Short TG, Leslie K, Chan MT, Campbell D, Frampton C, Myles P. Rationale and design of the balanced anesthesia study: a prospective randomized clinical trial of two levels of anesthetic depth on patient outcome after major surgery. Anesth Analg. 2015;121(2):357-365.
67. Sessler DI, Sigl JC, Kelley SD, et al. Hospital stay and mortality are increased in patients having a "triple low" of low blood pressure, low bispectral index, and low minimum alveolar concentration of volatile anesthesia. Periop Med. 2012;116(6):1195-1203.

68. Story DA. Postoperative mortality and complications. Best Pract Res Clin Anaesthesiol. 2011;25(3):319-327.

69. American Geriatrics Society Expert Panel on Postoperative Delirium in Older A. American Geriatrics Society abstracted clinical practice guideline for postoperative delirium in older adults. J Am Geriatr Soc. 2015;63(1):142-150.

70. Optimal perioperative management of the geriatric patient: best pracies guideline from ACS NSQIP/American Geriatrics Society. 2016; Available from: https:/www.facs.org/quality-programs/acs-nsqip/ geriatric-periop-guideline. Accessed April 26, 2018.

71. Riemen AH, Hutchison JD. The multidisciplinary management of hip fractures in older patients. Orthop Trauma. 2016;30(2):117-122.

72. Grigoryan KV, Javedan H, Rudolph JL. Orthogeriatric care models and outcomes in hip fracture patients: a systematic review and meta-analysis. J Orthop Trauma. 2014;28(3):e49-55.

73. Harari D, Hopper A, Dhesi J, Babic-Illman G, Lockwood L, Martin F. Proactive care of older people undergoing surgery (POPS): designing, embedding, evaluating and funding a comprehensive geriatric assessment service for older elective surgical patients. Age Ageing. 2007;36(2):190-196.

74. Kain ZN, Vakharia S, Garson L, et al. The perioperative surgical home as a future perioperative practice model. Anesth Analg. 2014;118(5):1126-1130.

75. Qiu C, Cannesson M, Morkos A, et al. Practice and outcomes of the perioperative surgical home in a California integrated delivery system. Anesth Analg. 2016;123(3):597-606.

76. Soffin EM, YaDeau JT. Enhanced recovery after surgery for primary hip and knee arthroplasty: a review of the evidence. Br J Anaesth. 2016;117(suppl 3):iii62-iii72.

77. Partridge JS, Harari D, Martin FC, Dhesi JK. The impact of pre-operative comprehensive geriatric assessment on postoperative outcomes in older patients undergoing scheduled surgery: a systematic review. Anaesthesia. 2014;69(Suppl 1):8-16.

78. Partridge JS, Harari D, Martin FC, et al. Randomized clinical trial of comprehensive geriatric assessment and optimization in vascular surgery. Br J Surg. 2017;104(6):679-687.

79. Chen CC, Li HC, Liang JT, et al. Effect of a Modified Hospital Elder Life Program on delirium and length of hospital stay in patients undergoing abdominal surgery: a cluster randomized clinical trial. JAMA Surg. 2017;152(9):827-834.

80. Gillis C, Li C, Lee L, et al. Prehabilitation versus rehabilitation: a randomized control trial in patients undergoing colorectal resection for cancer. Anesthesiology. 2014;121(5):937-947.

81. Carli F, Scheede-Bergdahl C. Prehabilitation to enhance perioperative care. Anesthesiol Clin. 2015;33(1):17-33.

82. Singh F, Newton RU, Galvao DA, Spry N, Baker MK. A systematic review of pre-surgical exercise intervention studies with cancer patients. Surg Oncol. 2013;22(2):92-104.

83. Theou O, Park GH, Garm A, Song X, Clarke B, Rockwood K. Reversing frailty levels in primary care using the CARES model. Can Geriatr J. 2017;20(3):105-111.

84. Salvi R, Meoli I, Cennamo A, et al. Preoperative high-intensity training in frail old patients undergoing pulmonary resection for NSCLC. Open Med (Wars). 2016;11(1):443-448.

85. Rumer KK, Saraswathula A, Melcher ML. Prehabilitation in our most frail surgical patients: are wearable fitness devices the next frontier? Curr Opin Organ Transplant. 2016;21(2):188-193.

86. Jakobsson J, Johnson MZ. Perioperative regional anaesthesia and postoperative longer-term outcomes. F1000Res. 2016;5: F1000 Faculty Rev-2501.

87. Popping DM, Elia N, Van Aken HK, et al. Impact of epidural analgesia on mortality and morbidity after surgery: systematic review and meta-analysis of randomized controlled trials. Ann Surg. 2014;259(6): 1056-1067. 
88. Guay J, Nishimori M, Kopp S. Epidural local anaesthetics versus opioidbased analgesic regimens for postoperative gastrointestinal paralysis, vomiting and pain after abdominal surgery. Cochrane Database Sys Rev. 2016;7:CD001893.

89. Schug SA, Palmer GM, Scott DA, Halliwell R, Trinca J. APM:SE Working Group of the Australian and New Zealand College of Anaesthetists and Faculty of Pain Medicine (2015), Acute Pain Management. Scientific Evidence (4th edition), ANZCA \& FPM, Melbourne. Available from: http://fpm.anzca.edu.au/documents/apmse4_2015_final. Accessed August 15, 2018.

90. Guay J, Parker MJ, Griffiths R, Kopp S. Peripheral nerve blocks for hip fractures. Cochrane Database Sys Rev. 2017;5:CD001159.

91. Chow WB, Rosenthal RA, Merkow RP, et al. Optimal preoperative assessment of the geriatric surgical patient: a best practices guideline from the American College of Surgeons National Surgical Quality Improvement Program and the American Geriatrics Society. J Am Coll Surg. 2012;215(4):453-466.

92. Knittel JG, Wildes TS. Preoperative assessment of geriatric patients. Anesthesiol Clin. 2016;34(1):171-183.
93. Kristensen SD, Knuuti J, Saraste A, et al. 2014 ESC/ESA guidelines on non-cardiac surgery: cardiovascular assessment and management: the Joint Task Force on non-cardiac surgery: cardiovascular assessment and management of the European Society of Cardiology (ESC) and the European Society of Anaesthesiology (ESA). Eur Heart J. 2014;35(35):2383-2431.

94. Fleisher LA, Beckman JA, Brown KA, et al. ACC/AHA 2007 guidelines on perioperative cardiovascular evaluation and care for noncardiac surgery: a report of the American College of Cardiology/American Heart Association Task Force on Practice Guidelines (Writing Committee to Revise the 2002 Guidelines on Perioperative Cardiovascular Evaluation for Noncardiac Surgery) developed in collaboration with the American Society of Echocardiography, American Society of Nuclear Cardiology, Heart Rhythm Society, Society of Cardiovascular Anesthesiologists, Society for Cardiovascular Angiography and Interventions, Society for Vascular Medicine and Biology, and Society for Vascular Surgery. J Am Coll Cardiol. 2007;50(17):e159-e241.
Local and Regional Anesthesia

\section{Publish your work in this journal}

Local and Regional Anesthesia is an international, peer-reviewed, open access journal publishing on the development, pharmacology, delivery and targeting and clinical use of local and regional anesthetics and analgesics. The journal is included in PubMed, and welcomes submitted papers covering original research, basic science, clinical studies,

\section{Dovepress}

reviews and evaluations, guidelines, expert opinion and commentary, case reports and extended reports. The manuscript management system is completely online and includes a very quick and fair peer-review system, which is all easy to use. Visit http://www.dovepress.com/ testimonials.php to read real quotes from published authors. 